\begin{tabular}{|c|l|}
\hline Title & Influence of implant rod curvature on sagittal correction of scoliosis deformity \\
\hline Author(s) & Salmingo, Remel A lingal an; Tadano, Shigeru; A be, Y uichiro; Ito, Manabu \\
\hline Citation & $\begin{array}{l}\text { Spine Journal, 14(8), 1432-1439 } \\
\text { https://doi.org/10.1016/.Spinee.2013.08.042 }\end{array}$ \\
\hline Issue Date & 201408 \\
\hline Doc URL & http://hdl.handle.net/2115/57457 \\
\hline Type & article (author version) \\
\hline File Information & HUA SCUP112614.pdf \\
\hline
\end{tabular}

Instructions for use 


\section{Influence of Implant Rod Curvature on Sagittal Correction of Scoliosis Deformity}

Remel Alingalan Salmingo, MEng

Laboratory of Biomechanical Design, Division of Human Mechanical Systems and Design, Graduate School of Engineering, Hokkaido University, North 13 West 8 Kita-Ku, Sapporo, JAPAN 060-8628

email: remel.salmingo@frontier.hokudai.ac.jp

Shigeru Tadano, $\mathrm{PhD}^{*}$

Division of Human Mechanical Systems and Design, Faculty of Engineering, Hokkaido

University, North 13 West 8 Kita-Ku, Sapporo, JAPAN 060-8628

email: tadano@eng.hokudai.ac.jp

Yuichiro Abe, MD, $\mathrm{PhD}$

Department of Orthopaedic Surgery, Eniwa Hospital, Kogane-Cho 2-1-1, Eniwa, JAPAN 0611449

email: menchi@athena.ocn.ne.jp

Manabu Ito, $\mathrm{MD}, \mathrm{PhD}$

Advanced Medicine for Spine and Spinal Cord Disorders, Graduate School of Medicine, Hokkaido University, North 15 West 7 Kita-Ku, Sapporo, JAPAN

060-8638

email: maito@med.hokudai.ac.jp

The device mentioned in this paper is approved by the FDA and corresponding national agency for this indication. No benefits in any form have been or will be received from a commercial party related directly or indirectly to the manuscript. This study was approved by the ethics committee of the university hospital.

* Address correspondence to:

Shigeru Tadano, PhD, Professor, Division of Human Mechanical Systems and Design, Faculty of Engineering, Hokkaido University, Sapporo, JAPAN 060-8628, Tel/Fax: +81117066405, email: tadano@eng.hokudai.ac.jp 


\section{Abstract}

BACKGROUND CONTEXT: In vivo implant rod deformation could alter the scoliosis sagittal correction. No previous study had investigated the influence of implant rod deformation on the sagittal deformity correction during scoliosis surgery.

PURPOSE: To analyze the changes of implant rod angle of curvature during surgery and establish its influence on sagittal correction of scoliosis deformity.

STUDY DESIGN: A retrospective analysis of the preoperative and postoperative implant rod geometry and angle of curvature was conducted.

PATIENT SAMPLE: Twenty adolescent idiopathic scoliosis patients underwent surgical operation. Average age at the time of operation was 14 years.

OUTCOME MEASURES: The preoperative and postoperative implant rod angle of curvature expressed in degrees was obtained for each patient.

METHODS: Two implant rods were attached to the concave and convex side of the spinal deformity. The preoperative implant rod geometry was measured before surgical implantation. The postoperative implant rod geometry after surgery was measured by Computed Tomography scanner. The implant rod angle of curvature at the sagittal plane was obtained from the implant rod geometry. The angle of curvature between the implant rod extreme ends was measured before implantation and after surgery. The sagittal curvature between the corresponding spinal levels of healthy adolescents obtained by previous studies was compared to the implant rod angle of curvature to evaluate the sagittal curve correction. The difference between the postoperative implant rod angle of curvature and normal spine sagittal curvature of the corresponding instrumented level was used to evaluate over or under correction of the sagittal deformity. 
RESULTS: The implant rods at the concave side of deformity of all patients were significantly deformed after surgery. The average degree of rod deformation $\Delta \theta$ at the concave and convex side was $15.8 \mathrm{deg}$. and $1.6 \mathrm{deg}$., respectively. The average preoperative and postoperative implant rod angle of curvature at the concave side was $33.6 \mathrm{deg}$. and 17.8 degrees, respectively. The average preoperative and postoperative implant rod angle of curvature at the convex side was 25.5 degrees and 23.9 degrees, respectively. A significant relationship was found between the degree of rod deformation and preoperative implant rod angle of curvature $(\mathrm{r}=0.60, \mathrm{p}<0.005)$. The implant rods at the convex side of all patients did not have significant deformation. The results indicate that the postoperative sagittal outcome could be predicted from the initial rod shape.

CONCLUSIONS: Changes in implant rod angle of curvature may lead to over or under correction of the sagittal curve. Rod deformation at the concave side suggests that corrective forces acting on that side are higher than the convex side.

\section{Keywords:}

Biomechanics, Rod Deformation, Implant Rod Curvature, Spine, Sagittal Correction, Adolescent Idiopathic Scoliosis

\section{Introduction}

Adolescent Idiopathic Scoliosis (AIS), a complex spinal pathology which arises from unknown etiology, is characterized as a three-dimensional deformity of the spine with vertebral rotation. The degree of severity of the scoliotic deformity is usually evaluated using the Cobb angle. The Cobb angle is defined as the maximum angle between two lines drawn 
parallel to the endplates of scoliotic vertebrae at the frontal plane. Scoliosis, however, is a complex deformity that needs to be assessed in three-dimensions. Several authors have developed mathematical expressions in order to measure the morphology of scoliotic spines in three dimensions as well as the rotation of vertebrae [1-3]. Since then, correction of scoliosis deformity in frontal, sagittal and axial planes has been widely studied [4-13]. Contrary to the frontal plane, sagittal plane deformity correction involves complex analysis of spine segments. Normally, the spine has thoracic kyphosis and lumbar lordosis curves. Neglecting to correct these curves could produce pain, intervertebral disc degeneration, boneimplant fracture, flat back and joint degeneration [14,15]. Many studies have investigated how to evaluate the sagittal correction. Generally, sagittal correction is measured using the thoracic sagittal kyphosis from the superior endplate of T4 to the inferior endplate of T12 while lumbar lordosis is measured from L1 to L5 [7]. In addition, more complex evaluation involves determination of sagittal balance. Although several authors proposed various indicators for measurement of sagittal balance, the Scoliosis Research Society (SRS) defined that normal sagittal alignment is achieved when the plumb line drawn at the center of the cervical vertebra C7 lies within $\pm 2 \mathrm{~cm}$ of the sacral promontory [16-20]. These methods advanced the evaluation of the sagittal plane correction. However, the fundamental limitation of visualizing the key anatomical landmarks still exists. Some studies reported inter and intra observer differences during measurements of sagittal spine curvatures [21-23]. The differences are due to the inherent anatomical variants that alters the normal spine symmetry from side-to-side [24,25]. In idiopathic scoliosis, asymmetry is primarily demonstrated by vertebral wedging. Failure to visualize the anatomical variants could lead to measurement errors. The postoperative lateral radiographs of previous studies show that the implant rod shape or curvature constitutes also the postoperative sagittal curve of the spine within the rod length, 
similar to Fig. 1(a) [26-32]. Hence, this indicates that the implant rod curvature could be also used for evaluation of the sagittal curve correction.

Until now, there is no consensus on what possible initial shape of rod could lead to a certain sagittal outcome. Optimal scoliosis surgical treatment is not always achieved due to the variability of surgeons' preferences, choice of approach, choice of instrumented level, surgeon experience and different correction objectives [33-34]. Several studies conducted biomechanical modeling to simulate various correction objectives, surgical steps and strategies. They estimated suitable surgical strategies for scoliosis patients. However, the results might be unrealistic because rod deformation was not considered in their analyses [3537]. The preoperative implant rod geometry was obtained only from postoperative data. Apparently, the reported magnitude of forces (in several hundred or even thousand Newtons) were high that should have deformed the implant rod during the surgical treatment [38]. Implant rod deformation could alter the sagittal alignment and consequently the clinical outcome. Thus, careful investigation of the changes of implant rod geometry is important to fully understand the biomechanics of scoliosis correction.

The objective of this study was to measure the preoperative and postoperative implant rod angle of curvature before surgical implantation and after surgery. The degree of rod deformation was evaluated as the difference of preoperative and postoperative implant rod angle of curvature. Relationship between the degree of rod deformation and preoperative implant rod angle of curvature was sought to establish whether it is possible to predict the postoperative outcome from the initial rod shape. Furthermore, the postoperative implant rod angle of curvature was used to evaluate the scoliosis sagittal correction, i.e. being over or under corrected. 


\section{Materials and Methods}

\subsection{Patients}

Twenty (20) scoliosis patients (average age: 14 years, range: 10-20 years) diagnosed as severe Adolescent Idiopathic Scoliosis (AIS) underwent surgical operation. A proper informed consent was obtained from all patients. All patients were operated after the approval of the university hospital research ethics committee on the use of human subjects. Six mm diameter (Ø6 mm) titanium implant rods and polyaxial pedicle screws (USS II Polyaxial, Synthes $\mathrm{GmbH}$, Switzerland) were used to correct the scoliosis deformity. The implant rod curvature and length vary with each patient. All rods were pre-bent only at a single plane. Rods and screws were surgically implanted following the double rod rotation technique procedure [32]. In this technique, two implant rods (i.e. for the concave and convex side of the deformity, Fig. 1(a)) were inserted into the polyaxial screw heads. The polyaxial screw heads remained unfastened until the completion of rod rotation allowing the rods to rotate and translate freely inside the screw head. A torque was applied to the rod rotating device to rotate simultaneously the rods (about 90 degrees); transferring the previous curvature of rod at the coronal plane to the sagittal plane.

\subsection{Implant Rod Deformation}

The implant rod angle of curvature was used to evaluate implant rod deformation. The implant rod angle of curvature at the corresponding extreme ends was measured using the preoperative and postoperative geometry of implant rod during the surgical operation, in Fig. 1(b). The preoperative and postoperative implant rod geometries for the concave and convex side of the 
deformity were obtained. The preoperative geometry of implant rod was measured from the actual implant rod used before surgical implantation. The postoperative implant rod geometry was obtained one week (maximum) after the surgical operation using CT scanner (Aquilion 64 CT Scan, Toshiba Medical Systems Corporation, Japan). The slice thickness was $0.5 \mathrm{~mm}$. The implant rod geometry was fitted using quintic polynomial function (minimum $\mathrm{R}^{2}$ value was set to 0.99 ) by the proposed numerical method [39]. Arbitrary points (7 points minimum) were selected for curve fitting. These points were obtained from the inferior to superior endpoint along the central axis of the implant rod. Medical image processing algorithm was programmed using computer software (MATLAB R2010b, Massachusetts, USA). The implant rod angle of curvature, i.e. the angle between two tangent vectors was computed using the first derivative of the quintic polynomial function evaluated at the implant rod ends $[1,2]$.

Measurements were repeated using three trials. The average of the three trials was obtained as the preoperative and postoperative implant rod angle of curvature, $\theta_{1}$ or $\theta_{2}$ of each patient, respectively (shown in Table 1). The postoperative implant rod angle of curvature $\theta_{2}$ was used to evaluate the sagittal curve correction of each scoliosis patient since it constitutes the sagittal curve of the spine after surgery. The difference between the preoperative and postoperative implant rod angle of curvature $\left(\theta_{1}-\theta_{2}\right)$ was referred to as the degree of rod deformation $\Delta \theta$. The relationship between the degree of rod deformation $\Delta \theta$ and preoperative implant rod angle of curvature $\theta_{1}$ was also investigated to establish whether it is possible to predict the postoperative outcome from the initial rod shape.

\subsection{Spine Sagittal Curvature}

The average thoracolumbar spine sagittal angle of curvature of healthy adolescents obtained by previous study was used to evaluate the sagittal correction [40]. Since the instrumented or 
fixation level of each patient differs from each other, the corresponding sagittal angle of curvature of each vertebra level was also determined. The angle of sagittal curvature of each vertebra level was approximated by the ratio of each height of vertebra [41]. The spine sagittal angle of curvature between the corresponding fixation level $\theta_{\mathrm{FL}}(\mathrm{FL}=$ most superior and inferior fixation level) of each patient is listed in Table 2. The spine sagittal angle of curvature of each patient is the same for the concave and convex side because the extreme fixation levels were the same for both sides of all scoliosis patients.

Ideal correction is attained when the postoperative implant rod angle of curvature $\theta_{2}$ equals to the sagittal angle of curvature $\theta_{\mathrm{FL}}$ of the healthy adolescents at the corresponding fixation level. Hence, with this approach, over or under correction of the sagittal curve could be determined by its difference. Over correction was defined when the postoperative implant rod angle of curvature $\theta_{2}$ is greater than the sagittal angle of curvature $\theta_{\mathrm{FL}}$ and vice versa.

\section{Results}

The preoperative $\theta_{1}$ and postoperative $\theta_{2}$ implant rod angle of curvatures at the concave and convex side of the deformity were obtained, listed in Table 1 and shown in Fig. 2. It can be noticed that the rod at the concave side was significantly deformed after the surgical treatment of scoliosis. For all patients, the results indicate that the rod curvature at the concave side tended to reduce. However, the rod at the convex side did not have significant deformation after the surgical treatment of scoliosis. The average preoperative and postoperative implant rod angle of curvature at the concave and convex side of all patients is shown in Fig. 3 . The average preoperative and postoperative implant rod angle of curvature at the concave side were 33.6 degrees (range 15.8 - 56.6 degrees) and 17.8 degrees (range 3.3 - 36.8 degrees), 
respectively. The average preoperative and postoperative implant rod angle of curvature at the convex side were 25.5 degrees (range 11.7 - 38.5 degrees) and 23.9 degrees (range 7.0 - 39.5 degrees), respectively.

A positive correlation was found between the degree of rod deformation $\Delta \theta$ and preoperative implant rod angle of curvature $\theta_{1}$ at the concave side $(r=0.60$,

$\mathrm{p}<0.005$ ), in Fig. 4. This means that the greater the implant rod was bent, the higher degree of rod deformation could be obtained. On the other hand, there was no significant correlation on the implant rods at the convex side $(r=0.26$,

$\mathrm{p}>0.05)$. Nevertheless, the implant rods at the convex side are predictable because it did not have significant deformation during scoliosis surgery. Thus, the results suggest that the postoperative implant rod curvature consequently the sagittal outcome can be predicted from the initial rod shape.

Ideal sagittal correction of scoliosis deformity is attained when the postoperative rod angle of curvature $\theta_{2}$ equals the sagittal curvature $\theta_{\mathrm{FL}}$ of the corresponding curvature of the extreme fixation level. The positive or negative difference between this parameter indicates over or under correction of the sagittal curvature, respectively. Figure 5 shows the difference between the postoperative rod angle of curvature $\theta_{2}$ at the concave and convex side of deformity, and sagittal curvature $\theta_{\mathrm{FL}}$ of the corresponding fixation level $\left(\theta_{2}-\theta_{\mathrm{FL}}\right)$. The upper region shows over correction and the lower region shows under correction. As a result, eight out of twenty patients (40\%) had 5 degrees or lesser difference (i.e. Patients 2, 4, 9, 10, 12, 13, 14, 16). Seven patients $(35 \%)$ had 10 degrees or lesser difference (i.e. Patient 3, 5, 6, 8, 11, 17, 18). Five patients had more than 10 degrees curvature difference (i.e. Patient 1, 7, 15, 19, 20). The result indicates that the implant rod curvature greatly influenced the sagittal curve correction because over or under correction might be obtained. 


\section{Discussion}

Many studies have reported the preservation of sagittal balance/alignment of the spine.

However, these studies were mainly focused on the postoperative results. The primary factor (i.e. initial rod shape) that is influencing the postoperative or sagittal outcome is not yet well studied. Indeed, until now, there is no consensus on what possible initial shape of rod could result to a certain sagittal outcome. Decision-making is always based on individual surgeons' preferences and experience [33-35]. In this study, although rod deformation occurred, we have found a positive trend between the degree of rod deformation $\Delta \theta$ and preoperative implant rod angle of curvature $\theta_{1}$ at the concave side. The significant relationship found in this study $(\mathrm{r}=$ $0.60, \mathrm{p}<0.005)$ suggests that prediction on what preoperative shape of rod could result into a certain sagittal curve is possible. Future studies involving more patients will further enhance the relationship and likewise establish the optimal rod shape for certain scoliosis patients. Visualizing the key anatomical landmarks and observer errors are the difficulties in assessing the postoperative sagittal alignment using planar radiographs. Intra and inter-observer studies were conducted to determine the accuracy and reliability of computer methods. Recently, digital radiographs enable users to adjust contrast and brightness. In effect, the anatomical landmarks can be highlighted which was not possible in conventional radiographs. Previous studies were successful in increasing the accuracy and reliability of sagittal profile measurement using computer-assisted programs [21,22]. However, the method needs many anatomical landmarks (i.e. requiring additional information input or computational time) which might be difficult to visualize in radiographs [42]. In addition, it is well understood that spinal deformities have inherent anatomical variants resulting to morphological asymmetry. 
The method presented here is relatively simple because it does not require any anatomical feature which might be obscured in digital radiographs.

The degree of rod deformation at the concave side indicates that the rods were experiencing higher corrective forces than those at the convex side. This could be explained by the axial rotation and displacement of vertebra during the morphological growth of AIS. Many authors have investigated the cause of axial rotation of vertebrae $[2,43,44]$. They found out that the lateral deviation of spine shifts the center of axial rotation of vertebra. The shift produces eccentric gravitational loads and moments causing the spinous process to rotate towards the concave side of the scoliosis deformity. As a consequence, the concave side of the deformity tends to displace anteriorly while posteriorly at the convex side. Thus, the translational and rotational displacement required for correction at the concave side is always higher than the convex side which results also to higher corrective forces at that side. These findings could be also due to the kinematics during simultaneous rotation of rods. From a mechanical point of view, if torsion occurs in a certain body, the moments/force at the center of rotation is always zero or minimum. It could be hypothesized that the center of rotation of vertebra during simultaneous rotation of rods might be located at around the convex side because the corrective forces acting on that side are negligible as indicated by the degree of rod deformation, shown in Fig. 6. Further study is required to validate this hypothesis through intraoperative measurement of three-dimensional spinal motion segments during scoliosis surgery.

The average physiological sagittal curvature of healthy adolescents obtained by previous studies was used to evaluate the sagittal correction, whether it was over and under corrected. It is well understood that interpersonal differences such as height, weight, age, sex and etc. affect the sagittal curvature of scoliosis or even normal subjects [40]. A more detailed analysis 
considering the interpersonal differences will further improve the assessment, i.e. using the implant rod curvature to evaluate the sagittal correction of scoliosis deformity.

This study presents the deformation behavior of titanium implant rods during correction of scoliosis deformities. Some stiffer spinal rod materials such as stainless steel and CobaltChrome rods (stiffest of the three) may exhibit lesser implant rod deformation and deliver higher corrective forces than the titanium rods. The stiffer implant rods may provide also greater degree of deformity correction during scoliosis surgery. However, stiffer rods could inhibit bone formation and remodeling because they take too much of the applied loads instead of the spine [45]. Likewise, from a mechanical point of view, stiffer rods induce higher corrective force which might be too high and could lead to the pullout of screw from the vertebra. Conversely, we have demonstrated in this study that the degree of deformity correction at the sagittal plane could be also attained or predicted as a function of the preoperative implant rod curvature not only the implant rod stiffness. A recent study has also showed that the magnitude of corrective forces acting on the deformed titanium rods during scoliosis surgery was within the safe level of pullout forces (i.e. $439 \mathrm{~N} \max$ ) [46]. In this study, we investigated whether we can estimate the postoperative implant rod angle of curvature from the initial implant rod shape. This was attained by measurement of the changes of implant rod geometry before implantation and after surgery (taken by postoperative CT). The postoperative CT was used because the two implant rods (i.e. rod at the concave and convex side) overlap each other at the sagittal plane, as shown in Fig. 1(a). Thus, we could not obtain the geometry of each implant rod using just planar sagittal radiograph. Conversely, CT scan can perform image segmentation and obtain the geometry of each rod at both sides postoperatively (Fig. 1(b)). In order to prevent variation in the technique, the same surgeons performed the same surgical technique in all cases. 


\section{Conclusions}

The present study showed the influence of implant rod curvature on sagittal correction of scoliosis deformity. Careful planning of the preoperative implant rod curvature is necessary to prevent over or under correction of scoliosis deformity. The positive correlation between the degree of rod deformation and preoperative implant rod curvature implies that the postoperative implant rod curvature or clinical outcome could be predicted. Furthermore, the deformation of rod indicated that the corrective forces required at the concave side are higher than the convex side of deformity.

\section{References}

1. Kanayama M, Tadano S, Kaneda K, Ukai T, Abumi K. A mathematical expression of three-dimensional configuration of the scoliotic spine. ASME Journal of Biomechanical Engineering 1996;118;247-52.

2. Tadano S, Kanayama M, Ukai T, Kaneda K. Morphological modeling and growth simulation of idiopathic scoliosis. In: Hayashi H, Ishikawa K, eds. Computational Biomechanics. Tokyo: Springer, 1996: 67-87.

3. Stokes I, Bigalow L, Moreland M. Three-dimensional spinal curvature in idiopathic scoliosis. Journal of Orthopaedic Research 1987; 5;102-13.

4. De Jonge T, Dubousset J, Illes T. Sagittal plane correction in idiopathic scoliosis. Spine 2002;27; 754-60.

5. Smith J. Adult deformity: management of sagittal plane deformity in revision adult spine surgery. Current Opinion in Orthopedics 2001;12; 206-15. 
6. Vialle R, Levassor N, Rillardon L, Templier A, Skalli W, Guigui P. Radiographic analysis of the sagittal alignment and balance of the spine in asymptomatic subjects. Journal of Bone and Joint Surgery 2005;87;260-7.

7. Lowenstein J, Matsumoto H, Vitale M, et al. Coronal and sagittal plane correction in adolescent idiopathic scoliosis: a comparison between all pedicle screw versus hybrid thoracic hook lumbar screw constructs. Spine 2007;32;448-52.

8. Rhee J, Bridwell K, Won D, Lenke L, Chotigavanichaya C, Hanson D. Sagittal plane analysis of adolescent idiopathic scoliosis: the effect of anterior versus posterior instrumentation. Spine 2002;27;2350-6.

9. Lee, S, Suk S, Chung E. Direct vertebral rotation: a new technique of three-dimensional deformity correction with segmental pedicle screw fixation in adolescent idiopathic scoliosis. Spine 2004;29;343-9.

10. Kim Y, Lenke L, Kim J, et al. Comparative analysis of pedicle screw versus hybrid instrumentation in posterior spinal fusion of adolescent idiopathic scoliosis. Spine $2006 ; 31 ; 291-8$

11. Delorme S, Labelle H, Aubin C, et al. A three-dimensional radiographic comparison of Cotrel-Dubousset and Colorado instrumentations for the correction of idiopathic scoliosis. Spine 2000;25;205-10.

12. Cheng J, Lebow R, Schmidt M, Spooner J. Rod derotation techniques for thoracolumbar spinal deformity. Neurosurgery 2008;63;149-56.

13. Storer S, Vitale M, Hyman J, Lee F, Choe J, Roye D. Correction of adolescent idiopathic scoliosis using thoracic pedicle screw fixation versus hook constructs. Journal of Pediatric Orthopedics 2005;25;415-9. 
14. Vrtovec T, Janssen M, Likar B, Castelein R, Viergever M, Pernus F. A review of methods for evaluating the quantitative parameters of sagittal pelvic alignment. Spine Journal $2012 ; 12 ; 433-46$.

15. Knott P, Mardjetko S, Techy F. The use of the T1 sagittal angle in predicting overall sagittal balance of the spine. Spine Journal 2010;10;994-8.

16. Ondra S, Marzouk S. Principles of sagittal plane deformity. In: Heary R, Albert T, eds. Spinal deformites: the essentials. New York: Thieme, 2007:59-65.

17. Legaye J, Duval-Beaupere G, Hecquet J, Marty C. Pelvic Incidence: A fundamental pelvic parameter for three-dimensional regulation of spinal sagittal curves. European Spine Journal 1998;7;99-103.

18. Roussouly P, Nnadi C. Sagittal plane deformity: an overview of interpretation and management. European Spine Journal 2010;19;1824-36.

19. Berthonnaud E, Dimnet J, Roussouly P, Labelle H. Analysis of the sagittal balance of the spine and pelvis using shape and orientation parameters. Journal of Spinal Disorders and Techniques 2005;18;40-7.

20. Boulay C, Tardieu C, Hecquet J, et al. Sagittal alignment of spine and pelvis Regulated by Pelvic Incidence: Standard Values and Prediction of Lordosis. European Spine Journal 2006;15;415-22.

21. Dimar J, Carreon L, Labelle H, et al. Intra- and inter-observer reliability of determining radiographic sagittal parameters of the spine and pelvis using a manual and a computerassisted methods. European Spine Journal 2008;17;1373-79.

22. Berthonnaud E, Labelle H, Roussouly P, Grimard G, Vaz G, Dimnet J. A variability study of computerized sagittal spinopelvic radiologic measurements of trunk balance. Journal of Spinal Disorders and Techniques 2005;18;66-71. 
23. Labelle H, Dansereau J, Bellefleur C, Jequier J. Variability of geometric measurements from three-dimensional reconstructions of scoliotic spines and rib cages. European Spine Journal 1995;4;88-94.

24. Liljenqvist U, Allkemper T, Hackenberg L, Link T, Steinbeck J, Halm H. Analysis of vertebral morphology in idiopathic scoliosis with use of magnetic resonance imaging and multiplanar reconstruction. Journal of Bone Joint Surgery Am 2002;84;359-68.

25. Rajwani T, Bagnall K, Lambert R, et al. Using magnetic resonance imaging to characterize pedicle asymmetry in both normal patients and patients with adolescent idiopathic scoliosis. Spine 2004;29;145-52.

26. Clement J, Chau E, Kimkpe C, Vallade M. Restoration of thoracic kyphosis by posterior instrumentation in adolescent idiopathic scoliosis: comparative radiographic analysis of two methods of reduction. Spine 2008;33;1579-87.

27. Asher M, Lai S, Burton D. Analysis of instrumentation/fusion survivorship without reoperation after primary posterior multiple anchor instrumentation and arthrodesis for idiopathic scoliosis. Spine Journal 2010;10;5-15.

28. Lowenstein J, Matsumoto H, Vitale M, et al. Coronal and sagittal plane correction in adolescent idiopathic scoliosis: a comparison between all pedicle screws versus hybrid thoracic hook lumbar screw constructs. Spine 2007;32;448-52.

29. Benli I, Ates B, Akalin S, Citak M, Kaya A, Alanay A. Minimum 10 years follow-up surgical results of adolescent idiopathic scoliosis patients treated with TSRH instrumentation. European Spine Journal 2007;16; 381-91.

30. Hwang S, Samdani A, Gressot L, et al. Effect of direct vertebral body derotation on the sagittal profile in adolescent idiopathic scoliosis. European Spine Journal 2012;21;31-9. 
31. Patel A, Schwab F, Ungar B, Farcy J, Lafage V. Analysis of sagittal plane deformity and correction. Current Orthopaedic Practice 2010;21;356-63.

32. Ito M, Abumi K, Kotani Y, et al. Simultaneous double rod rotation technique in posterior instrumentation surgery for correction of adolescent idiopathic scoliosis. Journal of Neurosurgery Spine 2010;12;293-300.

33. Majdouline Y, Aubin C, Sangole A, Labelle H. Computer simulation for the optimization of instrumentation strategies in adolescent idiopathic scoliosis. Medical and Biological Engineering and Computing 2009;47;1143-54.

34. Desroches G, Aubin C, Sucato D, Rivard C. Simulation of an anterior spine instrumentation in adolescent idiopathic scoliosis using a flexible multi-body model. Medical and Biological Engineering and Computing 2007;45;759-68.

35. Aubin C, Labelle H, Chevrefils C, Desroches G, Clin J, Biovin A. Preoperative planning simulator for spinal deformity surgeries. Spine 2008;33;2143-52.

36. Aubin C, Petit Y, Stokes I, Poulin F, Gardner-Morse M, Labelle H. Biomechanical modeling of posterior instrumentation of the scoliotic spine. Computer Methods in Biomechanics and Biomedical Engineering 2003;6;27-32.

37. Lafon Y, Lafage V, Dubousset J, Skalli W. Intraoperative three-dimensional correction during rod rotation technique. Spine 2009;34;512-9.

38. Salmingo R, Tadano S, Fujisaki K, Abe Y, Ito M. Corrective force analysis for scoliosis from implant rod deformation. Clinical Biomechanics, 2012;27;545-50.

39. Salmingo R, Tadano S, Fujisaki K, Abe Y, Ito M. A simple method for in vivo measurement of implant rod three-dimensional geometry during scoliosis surgery. ASME Journal of Biomechanical Engineering 2012;134;054502. 
40. Mac-Thiong J, Labelle H, Berthonnaud E, Betz R, Roussouly P. Sagittal spinopelvic balance in normal children and adolescents. European Spine Journal 2007;16;227-34.

41. Kunkel M, Herkommer A, Reinehr M, Bockers T, Wilke H. Morphometric analysis of the relationships between intervertebral disc and vertebral body heights: an anatomical and radiographic study of the thoracic spine. Anatomy 2011;219;375-87.

42. Anderst W, Vaidya R, Tashman S. A technique to measure three-dimensional in vivo rotation of fused and adjacent lumbar vertebrae. Spine Journal 2008;8:991-7.

43. Stokes I. Axial rotation component of thoracic scoliosis. Journal of Orthopaedic Research $1989 ; 7 ; 702-8$.

44. White A, Panjabi M. Clinical biomechanics of the spine. Philadelphia: Lippincott Company, 1978:91-114.

45. Gummerson N, Millner P. Spinal fusion for scoliosis, clinical decision-making and choice of approach and devices. Skeletal Radiology 2010;39:939-42.

46. Salmingo R, Tadano S, Fujisaki K, Abe Y, Ito M. Relationship of forces acting on implant rods and degree of scoliosis correction. Clinical Biomechanics, http://dx.doi.org/10.1016/j.clinbiomech.2012.12.001. 
Table 1 Implant rod angle of curvature at the concave and convex side of deformity of each patient.

\begin{tabular}{|c|c|c|c|c|c|c|}
\hline \multirow[t]{2}{*}{$\overline{\text { Patient }}$} & \multicolumn{3}{|c|}{ Concave side (deg.) } & \multicolumn{3}{|c|}{ Convex side (deg.) } \\
\hline & $\overline{\theta_{1}}$ & $\theta_{2}$ & $\Delta \theta$ & $\overline{\theta_{1}}$ & $\theta_{2}$ & $\Delta \theta$ \\
\hline$\overline{1}$ & 44.6 & 25.1 & 19.5 & 35.2 & 33.4 & 1.9 \\
\hline 2 & 38.1 & 30.6 & 7.6 & 20.3 & 23.3 & -3.0 \\
\hline 3 & 43.4 & 19.7 & 23.7 & 34.7 & 27.3 & 7.4 \\
\hline 4 & 18.5 & 10.6 & 8.0 & 20.6 & 16.3 & 4.3 \\
\hline 5 & 19.1 & 5.0 & 14.1 & 19.7 & 24.6 & -4.9 \\
\hline 6 & 28.3 & 13.6 & 14.7 & 26.3 & 23.5 & 2.7 \\
\hline 7 & 46.5 & 9.7 & 36.8 & 26.2 & 23.4 & 2.9 \\
\hline 8 & 56.6 & 35.3 & 21.3 & 34.4 & 34.8 & -0.4 \\
\hline 9 & 18.0 & 6.1 & 11.9 & 11.7 & 7.0 & 4.7 \\
\hline 10 & 37.0 & 18.3 & 18.7 & 25.3 & 26.6 & -1.3 \\
\hline 11 & 15.8 & 3.3 & 12.5 & 15.8 & 19.8 & -4.0 \\
\hline 12 & 28.8 & 13.6 & 15.3 & 20.1 & 14.2 & 5.9 \\
\hline 13 & 18.1 & 5.7 & 12.4 & 12.8 & 14.5 & -1.7 \\
\hline 14 & 45.6 & 36.8 & 8.8 & 29.6 & 28.4 & 1.2 \\
\hline 15 & 22.3 & 19.3 & 3.0 & 22.1 & 18.0 & 4.1 \\
\hline 16 & 25.9 & 12.5 & 13.4 & 20.5 & 15.5 & 5.0 \\
\hline 17 & 42.9 & 18.1 & 24.9 & 36.5 & 27.1 & 9.4 \\
\hline 18 & 48.6 & 29.0 & 19.6 & 38.5 & 33.0 & 5.5 \\
\hline 19 & 33.2 & 20.2 & 13.0 & 24.2 & 28.1 & -3.9 \\
\hline 20 & 41.3 & 23.4 & 17.9 & 34.7 & 39.5 & -4.8 \\
\hline
\end{tabular}

$\theta_{1}$ Preoperative rod angle of curvature

$\theta_{2}$ Postoperative rod angle of curvature

$\Delta \theta$ Degree of rod deformation 
Table 2 Normal spine sagittal curvature at the corresponding fixation level obtained from previous studies $[40,41]$.

\begin{tabular}{ccc}
\hline Patient & $\begin{array}{c}\text { Fixation Level } \\
\text { (FL) }\end{array}$ & $\begin{array}{c}\text { Spine Sagittal Curve } \\
\theta_{\text {FL }} \text { (deg.) }\end{array}$ \\
\hline 1 & T5-L2 & 12.3 \\
2 & T6-T12 & 27.7 \\
3 & T6-L1 & 18.4 \\
4 & T2-L3 & 12.5 \\
5 & T2-L3 & 12.5 \\
6 & T6-L2 & 8.9 \\
7 & T5-L1 & 21.8 \\
8 & T2-T12 & 41.1 \\
9 & T5-L3 & 2.6 \\
10 & T5-L1 & 21.8 \\
11 & T4-L3 & 6.0 \\
12 & T6-L2 & 8.9 \\
13 & T3-L3 & 9.4 \\
14 & T5-T12 & 31.1 \\
15 & T2-T11 & 36.6 \\
16 & T5-L2 & 12.3 \\
17 & T6-T12 & 27.7 \\
18 & T4-L1 & 25.3 \\
19 & T4-T12 & 34.5 \\
20 & T2-T11 & 36.6 \\
\hline
\end{tabular}




\section{List of Figures}

Fig. 1(a) Frontal and sagittal radiographs of spine after surgery (b) Preoperative and postoperative implant rod angle of curvature.

Fig. 2 Preoperative and postoperative implant rod angle of curvature at concave and convex side of deformity.

Fig. 3 Average implant rod angle of curvature measured preoperatively and postoperatively.

Fig. 4 Relationship between the preoperative implant rod angle of curvature and degree of rod deformation at the concave side.

Fig. 5 Difference between the postoperative implant rod angle of curvature $\theta_{2}$ and physiological sagittal curvature $\theta_{\mathrm{FL}}$ at the corresponding fixation level.

Fig. 6 Rotation of the vertebra due to scoliosis deformity. 
Fig. 1

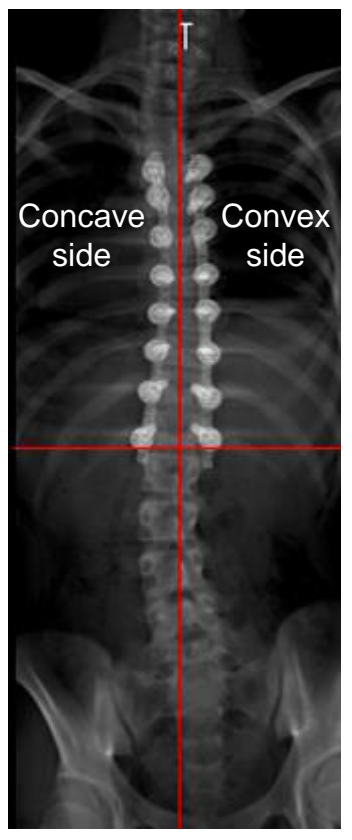

Frontal radiograph

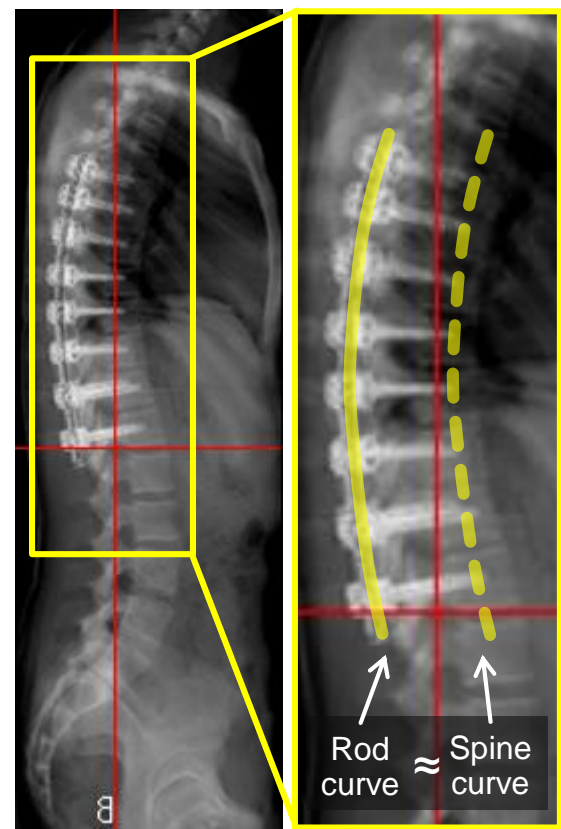

Sagittal radiograph

(a)

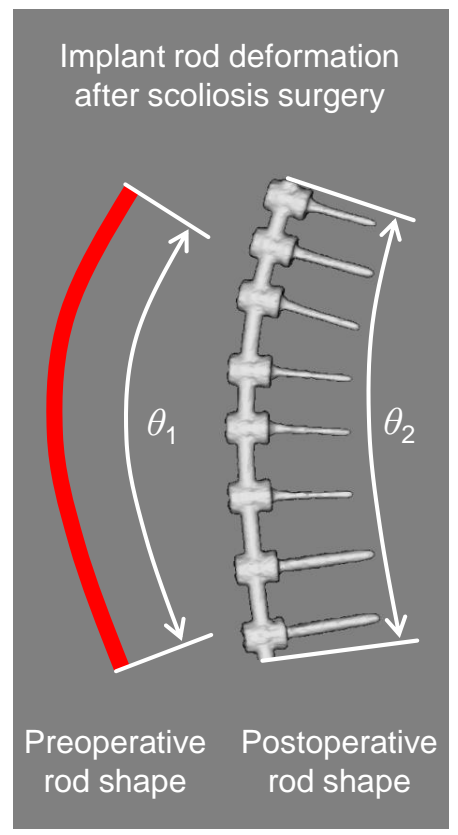

Implant rod angle of curvature

(b) 
Fig. 2

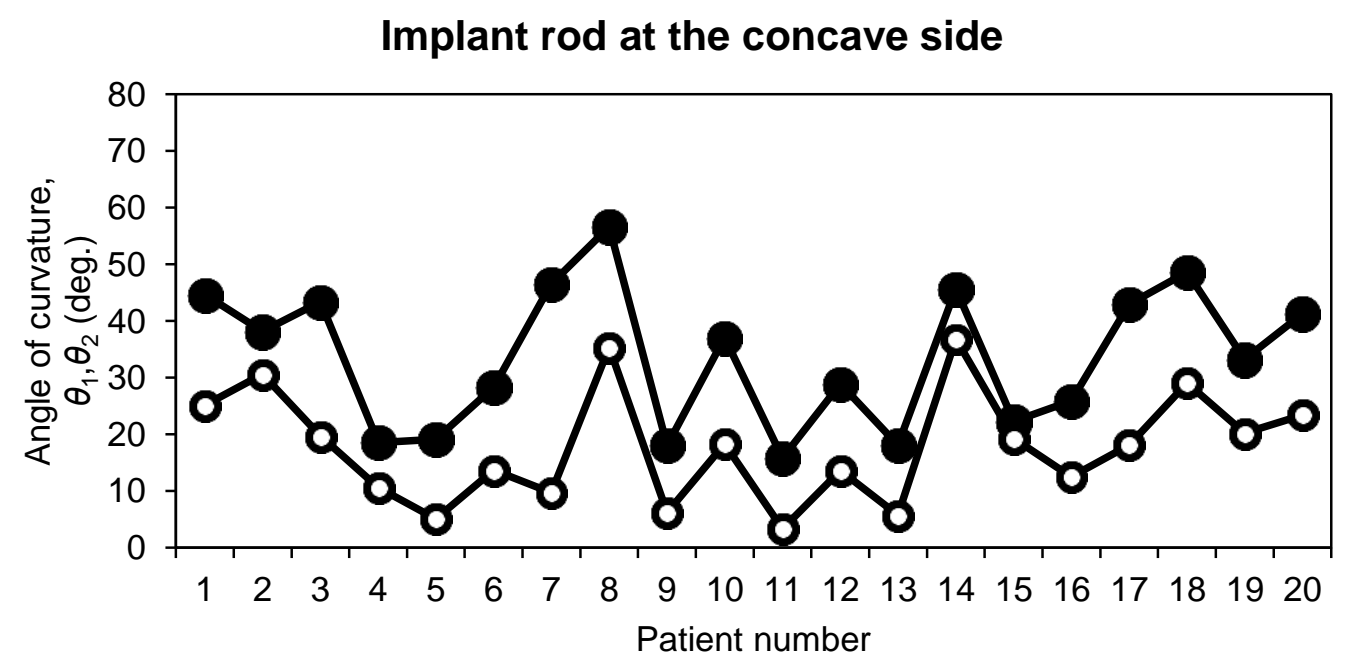

-Preoperative, $\theta_{1} \quad$ - Postoperative, $\theta_{2}$

Implant rod at the convex side

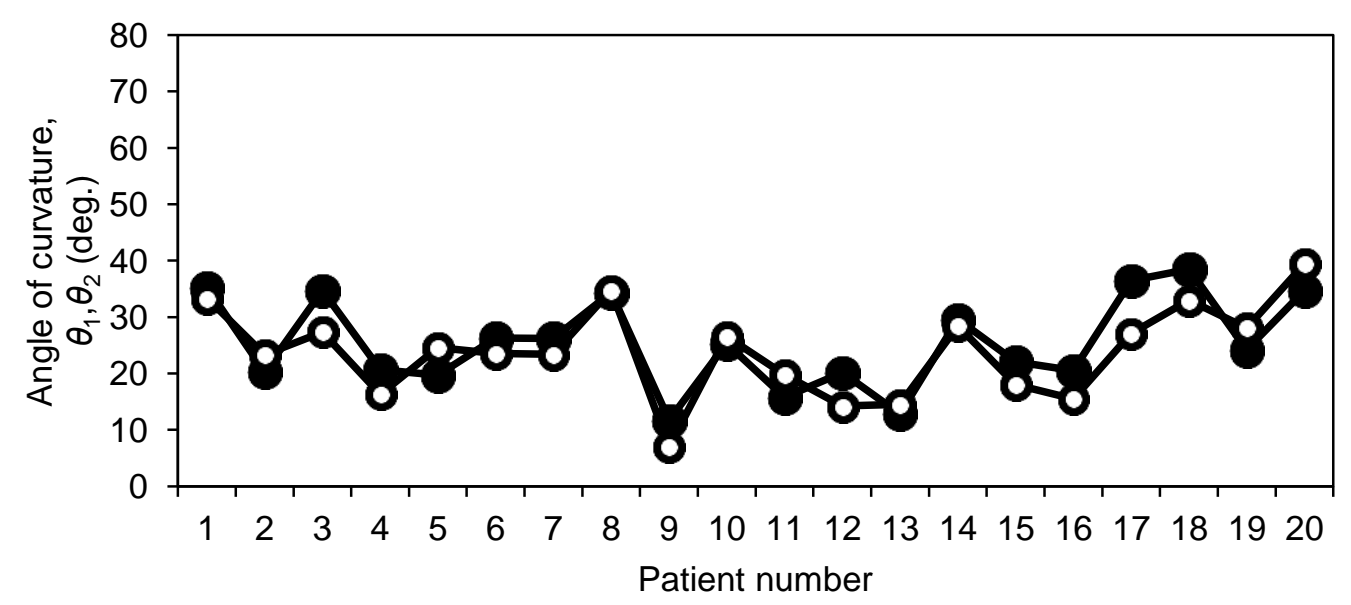

-Preoperative, $\theta_{1} \quad$ - Postoperative, $\theta_{2}$ 
Fig. 3

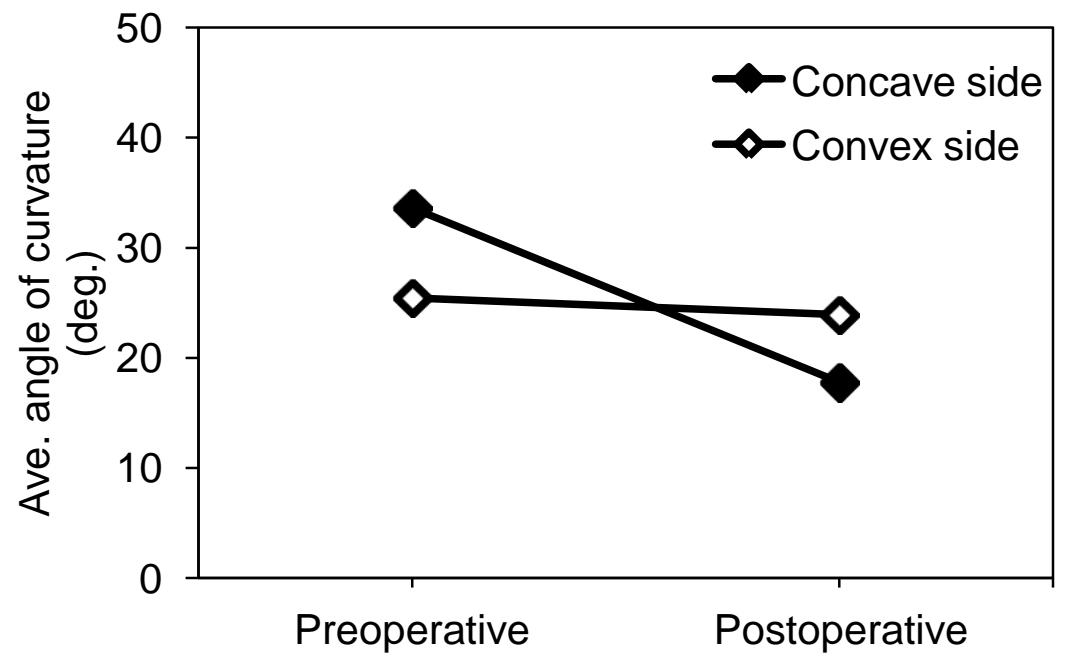

Page | 24 
Fig. 4

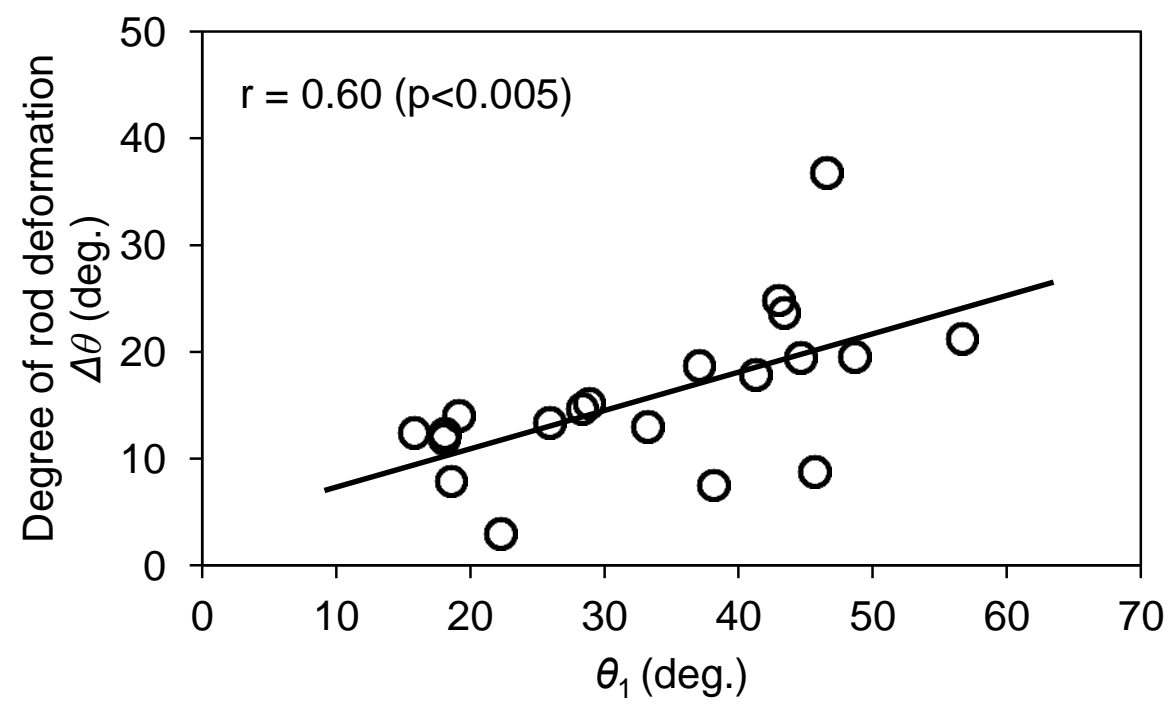


Fig. 5

Implant rod at the concave side

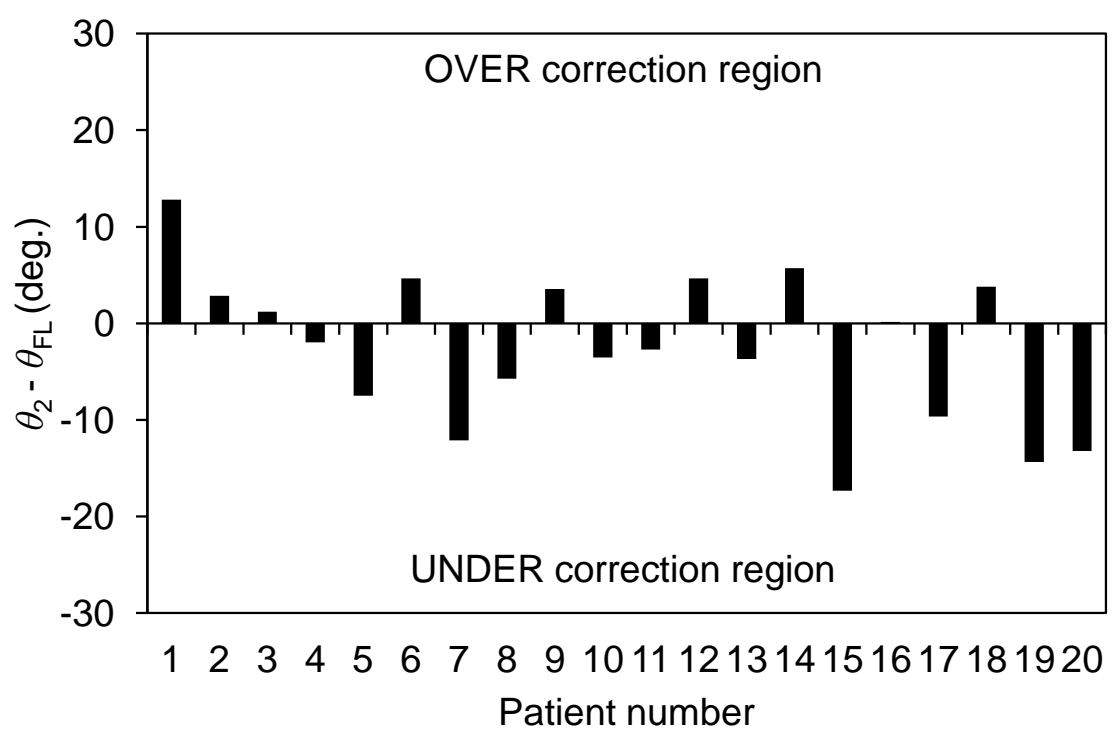

Implant rod at the convex side

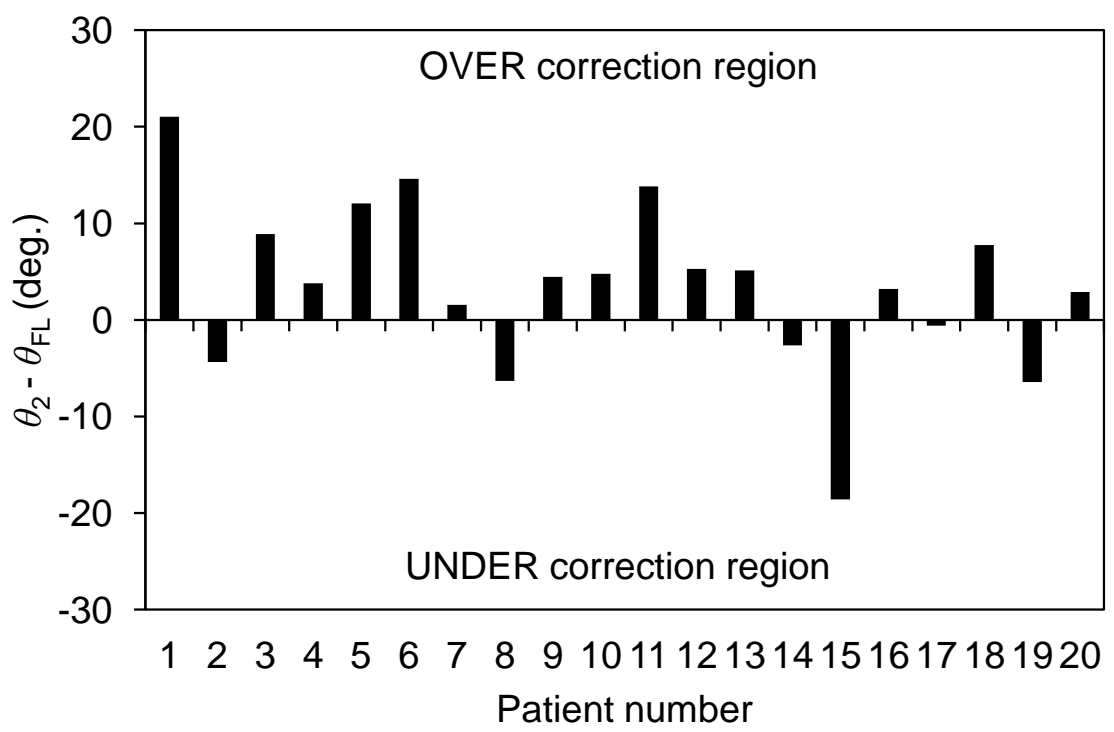


Fig. 6

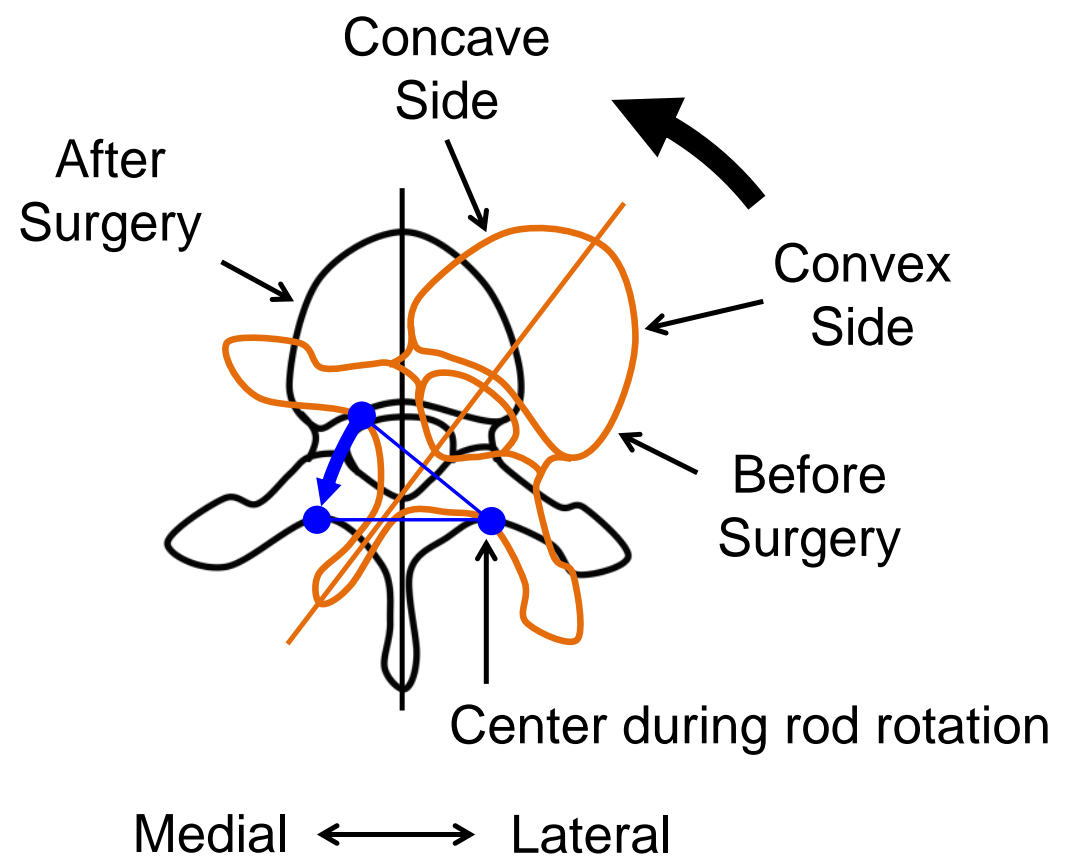

